Deutsches Zentrum

DLR für Luft- und Raumfahrt e.V.

in der Helmholtz-Gemeinschaft

Originally published as:

Steigenberger P., Montenbruck O., Hugentobler U. (2015): GIOVE-B Solar Radiation Pressure Modeling for Precise Orbit Determination, Advances in Space Research, Vol. 55, No. 5, pp. 1422-1431, DOI: 10.1016/j.asr.2014.12.009

The original publication is available at http://www.elsevier.com

(c) 2015. This manuscript version is made available under the CC-BY-NC-ND 4.0 license http://creativecommons.org/licenses/by-nc-nd/4.0/

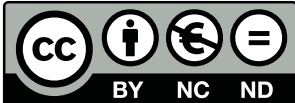




\title{
GIOVE-B Solar Radiation Pressure Modeling for Precise Orbit Determination
}

\author{
Peter Steigenberger ${ }^{\mathrm{a}}$, Oliver Montenbruck ${ }^{\mathrm{a}}$ and Urs Hugentobler ${ }^{\mathrm{b}}$ \\ ${ }^{a}$ German Space Operations Center (GSOC), Deutsches Zentrum für Luft-und Raumfahrt (DLR), Münchener Straße 20, \\ D-82234 Weßling, Germany \\ ${ }^{\mathrm{b}}$ Institut für Astronomische und Physikalische Geodäsie, Technische Universität München, Arcisstraße 21, D-80290 München, \\ Germany
}

\begin{abstract}
Previous studies have identified systematic errors in the orbit and clock estimates of the GIOVE and Galileo IOV satellites in the order of $\pm 20 \mathrm{~cm}$. These errors are visible as periodic variations in the Satellite Laser Ranging (SLR) and clock residuals. For IOV, these variations could be attributed to the contribution of a stretched satellite body and it was shown that a simple a priori box model for the solar radiation pressure can significantly reduce these errors. GIOVE-B has similar dimensions as the IOV satellites but its orientation is different: for GIOVE-B the narrow side of the satellite points to the Earth rather than the longitudinal side. In addition, an extra plate carrying, amongst others, the laser retro reflector array is mounted on the spacecraft introducing shadowing effects. These features are considered with a simple boxplate model. This model reduces the periodic clock errors and the SLR residual RMS of GIOVE-B by a factor of two. Most importantly, the box-plate model reduces the SLR offset from $11 \mathrm{~cm}$ to less than $1 \mathrm{~cm}$. The largest part of this reduction comes from considering the plate and its shadowing effects.
\end{abstract}

Keywords: GNSS; Galileo; Satellite Orbits; Solar radiation pressure; box-plate model

\section{Introduction}

GIOVE-B is the second test satellite of the European Global Navigation Satellite System (GNSS) Galileo. It was launched in 2008 as part of the Galileo In-Orbit Validation Element (GIOVE) and joined GIOVE-A that was launched three years earlier (Benedicto et al., 2006). The installation of the operational space segment of Galileo started with four In-Orbit Validation (IOV) satellites launched in 2011 and 2012, respectively, and the first launch of a pair of Full Operational Capability (FOC) satellites in August 2014.

Due to a very restricted data policy, early analysis of GIOVE data were limited to the European Space Agency (ESA) and its contractors (García et al., 2008; Hidalgo et al., 2009; Kirchner et al., 2009; Schönemann et al., 2007) based on the Galileo Experimental Sensor Station (GESS, Giraud et al., 2009) network. In general, the dif- ferent authors achieved a few decimeter orbit accuracy. In 2009, Deutsches Zentrum für Luft- und Raumfahrt (DLR) and Bundesamt für Kartographie und Geodäsie (BKG) initiated the Cooperative Network for GIOVE Observation (CONGO, Montenbruck et al., 2011) to foster the collection of tracking data of these satellites and their analysis. Based on this network, Steigenberger et al. (2011) could also achieve a few decimeter orbit accuracy for GIOVE-B.

Systematic errors in the GIOVE-B satellite clock estimates as well as in the Satellite Laser Ranging (SLR) residuals with a peak-to-peak amplitude of up to $40 \mathrm{~cm}$ were identified by Svehla et al. (2010) and further discussed in Montenbruck et al. (2012). Figure 1 illustrates these effects for a data arc of one week. Due to the orbit height of GNSS satellites, SLR residuals mainly reflect radial orbit errors. These, as well as the clock residuals, exhibit periodic variations at the orbit frequency of $14 \mathrm{~h}$ indicating the presence of systematic orbit modeling errors. Analyses in the framework of the Multi-GNSS Experiment (MGEX) of the International GNSS Service (IGS, Dow et al., 2009) showed the presence of such systematic effects also in the products of different analysis centers for the Galileo IOV satellites (Prange et al., 2014; Steigenberger et al., 2014; Uhlemann et al., 2014). The dependence of the peak-topeak amplitude of SLR and clock residuals on the elevation of the Sun above the orbital plane $\beta$ suggested deficiencies in the modeling of the solar radiation pressure (SRP) as a possible origin.

Hackel et al. (2014) could demonstrate that the systematic orbit and clock errors can be mitigated by a combination of the microwave GNSS observations with optic SLR measurements. Steigenberger et al. (2014) developed a simple empirical clock correction model that partly compensates these errors. However, these approaches did not deal with the source of these systematic errors but just mitigated their order of magnitude by including additional measurements or applying an a posteriori correction model. Recently, Montenbruck et al. (2014) identified the specific shape of the Galileo IOV satellites as the cause for these systematic effects. Whereas the GPS satellites have an essentially cubic body, the GIOVE-B and Galileo IOV spacecraft (see Fig. 2) have a shape like a telephone box introducing additional accelerations due to SRP that have not been considered so far. Montenbruck et al. (2014) 


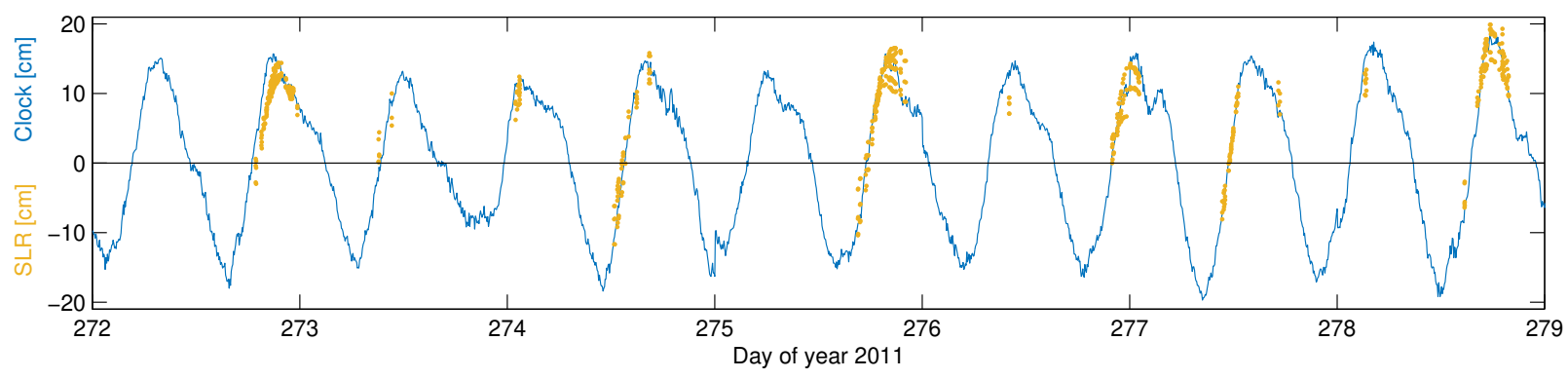

Fig. 1 Time series of GIOVE-B SLR and clock residuals. A mean bias has been removed from the SLR residuals whereas the sign of the clock residuals has been inverted in order to correspond to radial orbit errors and a 2nd order polynomial has been removed.

developed a simple a priori box model based on the accelerations acting on different surfaces of the satellite. By applying this model, they could reduce the systematic errors by a factor of about four.

The size of the Galileo IOV satellites and GIOVE-B is quite similar. However, the orientation of the spacecraft is different: the navigation antenna that has to point towards the Earth is mounted on the narrow side of the satellite for GIOVE-B whereas it is mounted on the longitudinal side for Galileo IOV and FOC. In addition, a plate carrying the GIOVE-B laser retroreflector array (LRA), an S-band antenna, and a Sun sensor introduces a more complex structure, see Fig. 2.

The GIOVE-B spacecraft is introduced in Sec. 2 and a simple box-plate model including shadowing effects is developed. The data, modeling, and parameter estimation options for the GNSS data processing are discussed in Sec. 3. Three different GNSS solutions have been computed in order to evaluate the impact of the a priori model: a reference solution without a priori model and two solutions with a box-only and a box-plate a priori model. These solutions provide the basis for the model evaluation in Sec. 4 as regards radiation pressure parameters, SLR, and clock residuals.

\section{Solar Radiation Pressure Modeling}

A commonly used model to account for SRP acting on GNSS satellites is the model of Beutler et al. (1994), also known as CODE model. The SRP accelerations are expressed in a Sun-oriented $D Y B$ frame with $D$ pointing from the satellite to the Sun, $Y$ along the solar panel axis, and $B=D \times Y$. In each direction, one constant term (index 0 ) and two harmonic terms (indices $C$ and $S$ ) are considered. Usually, only the five parameters $D_{0}, Y_{0}, B_{0}, B_{C}$, and $B_{S}$ are estimated. This model provides a good performance for the cubic GPS satellites but partly fails for the stretched GIOVE-B and Galileo satellites introducing systematic errors at the orbit frequency. Therefore, Montenbruck et al. (2014) applied a simple a priori box model taking into account the shape of these satellites. The general form of this model considers absorption plus diffuse reflection $(\alpha \delta)$ and specular reflection $(\rho)$ of the SRP acting on the $+z,-z$, and $+x$ surface of the satellite (the other surfaces are not illuminated if the satellite maintains nominal attitude). For a more detailed discussion on SRP modeling see Rodriguez-Solano et al. (2012) and Montenbruck et al. (2014).
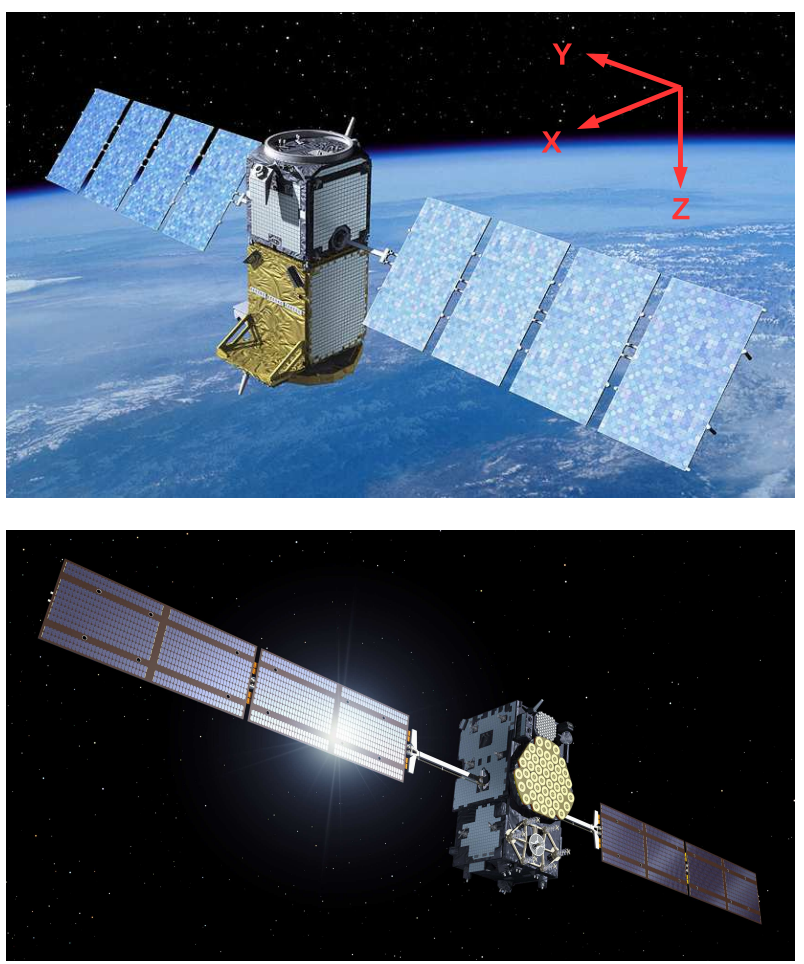

Fig. 2 Top: Artist's view of the GIOVE-B spacecraft (Image: ESA). The orientation of the spacecraft-fixed coordinate system follows Kouba and Héroux (2001) with the $z$-axis pointing to the Earth, the $y$-axis along the solar panel axis and $x=y \times z$ pointing into the hemisphere containing the Sun. The plate mentioned in the text can be seen in the lower left part of the figure pointing in the $+x$-direction. Bottom: Artist's view of a Galileo IOV spacecraft (Image: ESA). In contrast to GIOVE-B, the Earth-pointing navigation antenna is mounted on the long side of the cuboid-shaped satellite body.

\subsection{The GIOVE-B Spacecraft}

The GIOVE-B spacecraft shown in Fig. 2 was manufactured by EADS Astrium (Malik et al., 2009), launched on 27 April 2008, and started signal transmission on 7 May 2008 (Gao et al., 2008). Its orbit has an inclination of $56^{\circ}$ and a semimajor axis of $29,545 \mathrm{~km}$ resulting in a time of revolution of about $14 \mathrm{~h}$. After more than four years of operations and well beyond its designated lifetime of two 


\begin{tabular}{cccc}
\hline$m_{l}$ & $530 \mathrm{~kg}$ & $m_{a}$ & $509 \mathrm{~kg}$ \\
\hline$l_{x}$ & $0.95+0.5 \mathrm{~m}$ & $A_{x}$ & $2.3 \mathrm{~m}^{2}$ \\
$l_{y}$ & $0.95 \mathrm{~m}$ & $A_{y}$ & $2.3 \mathrm{~m}^{2}$ \\
$l_{z}$ & $2.4 \mathrm{~m}$ & $A_{z}$ & $1.7 \mathrm{~m}^{2}$ \\
\hline$\alpha \delta$ & 0.9 & $\rho$ & 0.1 \\
\hline
\end{tabular}

Table 1 Reference values of the satellite mass, dimensions, and optical properties of GIOVE-B. $m_{l}$ is the launch mass, $m_{a}$ the assumed mass in 2011, $l_{i}$ with $i=x, y, z$ stands for the length in each coordinate direction and $A_{i}$ for the corresponding surface areas. The optical properties are separated into an absorption plus diffuse reflection term $\alpha \delta$ and a specular reflection term $\rho$.

years, GIOVE-B was officially decommissioned on 23 July 2012 (GPS World, 2014) and moved to a graveyard orbit. A comprehensive summary of the GIOVE mission results is given in ESA (2011).

GIOVE-B's primary mission goals were securing of the frequency filing, testing of Galileo-specific payload like the first passive Hydrogen maser (PHM) in space, characterization of the orbit radiation conditions, and early GNSS signal experimentation (Robertson et al., 2009). GIOVE-B is equipped with one PHM (Ostillio et al., 2009) and two Rubidium (Rb, Droz et al., 2010) clocks providing a stable reference for generation of the navigation signals. To allow for an independent validation of the orbit determination results, GIOVE-B is equipped with a LRA (Dell'Agnello et al., 2011) for satellite laser ranging. LRA offset parameters are given in Zandbergen and Navarro (2008). Please note that the orientation of the spacecraft-fixed coordinate system in Fig. 2 follows the IGS convention ( $x$-axis pointing to the hemisphere containing the Sun, Kouba and Héroux, 2001) whereas the $x$ - and $y$-axis of Zandbergen and Navarro (2008) are mirrored w.r.t. this convention.

Table 1 lists coarse dimensions and the mass of GIOVE-B based on Robertson et al. (2009) and ESA (2011). The current mass $m_{a}$ is deduced from a launch mass of $530 \mathrm{~kg}$ and the assumption that $3 / 4$ of the initial amount of $28 \mathrm{~kg}$ of hydrazine have already been burned prior to the analysis period of this paper in 2011. The dimension in $x$-direction accounts for the satellite body $(0.95 \mathrm{~m})$ and the plate, which is assumed to have a width of $0.5 \mathrm{~m}$. The area in $z$-direction $A_{z}$ is slightly larger than $l_{x} \cdot l_{y}$ due to the excess length of the antenna panel w.r.t. the satellite body, see Fig. 2.

Figure 2 also shows that the satellite body is covered with a golden type of multilayer insulation (MLI) and solar cells. According to Rodriguez-Solano et al. (2012) optical properties for the golden MLI of GPS satellites are $\alpha \delta=0.9$ and $\rho=0.1$ whereas they vary for different types of solar cells with $\alpha \delta \approx 0.75 \ldots 0.8$ and $\rho \approx 0.25 \ldots 0.2$. Due to the unknown type and dimensions of the solar cells covering the satellite body, the golden MLI properties are used for the whole satellite body.

\subsection{GIOVE-B Box-Plate Model}

To account for the different shape of the Galileo IOV satellites compared to GPS, Montenbruck et al. (2014) added an a priori box model to the 5-parameter CODE model.
This model is based on characteristic accelerations $a_{i}$ due to absorption plus diffuse reflection $(\alpha \delta)$ and specular reflection $(\rho)$ of individual satellite surfaces $i$

$$
\begin{aligned}
a_{i}^{\alpha \delta} & =\frac{\Phi_{0}}{m \cdot c} \cdot A_{i} \cdot\left(\alpha_{i}+\delta_{i}\right) \\
a_{i}^{\rho} & =\frac{\Phi_{0}}{m \cdot c} \cdot A_{i} \cdot \rho_{i}
\end{aligned}
$$

with

$$
\begin{array}{ll}
\Phi_{0} & \text { solar flux at } 1 \mathrm{AU} \\
m & \text { spacecraft mass } \\
c & \text { vacuum speed of light } \\
A_{i} & \text { area of surface } i \\
\alpha_{i}+\delta_{i} & \text { absorption plus diffuse reflection } \\
& \text { coefficient of surface } i \\
\rho_{i} & \text { specular reflection coefficient of surface } i
\end{array}
$$

Assuming a nominal yaw-steering attitude law (BarSever, 1996), only the $+z,-z$, and $+x$ surfaces are illuminated by the Sun and have to be considered. The solar panels are not modeled as their effect is fully covered by the estimation of the direct SRP parameter $D_{0}$.

For practical applications, it is sufficient to consider only absorption plus diffuse reflection and to ignore specular reflection as well as an asymmetry of the $\pm z$-surfaces. Following Montenbruck et al. (2014), the simplified version of the box model in the $D Y B$ frame for a given Sun-satelliteEarth angle $\varepsilon$ then reads:

$$
\begin{aligned}
a_{\text {box }, D}= & -a_{C} \cdot\left(|\cos \varepsilon|+\sin \varepsilon+\frac{2}{3}\right) \\
& -a_{S} \cdot\left(|\cos \varepsilon|-\sin \varepsilon-\frac{4}{3} \sin ^{2} \varepsilon+\frac{2}{3}\right) \\
a_{\text {box }, B=}^{=} & -\frac{4}{3} a_{S} \cdot(\cos \varepsilon \sin \varepsilon) .
\end{aligned}
$$

Here,

$$
a_{C}=\frac{1}{2}\left(a_{z}+a_{+x}\right)
$$

and

$$
a_{S}=\frac{1}{2}\left(a_{z}-a_{+x}\right)
$$

denote the contributions of a pure cube and a stretching of a cuboid, respectively, which can be computed from the accelerations of the mean $z$-surface $a_{z}$ and the $+x$-surface $a_{+x}$. The acceleration in Sun-direction is composed of two contributions proportional to $a_{C}$ and $a_{S}$, respectively, which exhibit distinct and linearly independent dependencies on the Sun elongation. The $B$-component, in contrast, vanishes for a purely cubic shape and depends only on the stretch parameter $a_{S}$.

For GIOVE-B, an additional plate extending the $+z$ surface towards the $+x$ side of the spacecraft body (see Fig. 2) has to be considered. This plate not only increases the effective $\pm z$ cross section but also introduces notable shadowing effects that need to be considered in the radiation pressure modeling. Figure 3 illustrates the shadowing of the $+x$-surface by this plate. The $+x$-surface is completely in the shadow for

$$
\varepsilon<\arctan \frac{l_{p}}{l_{z}} .
$$




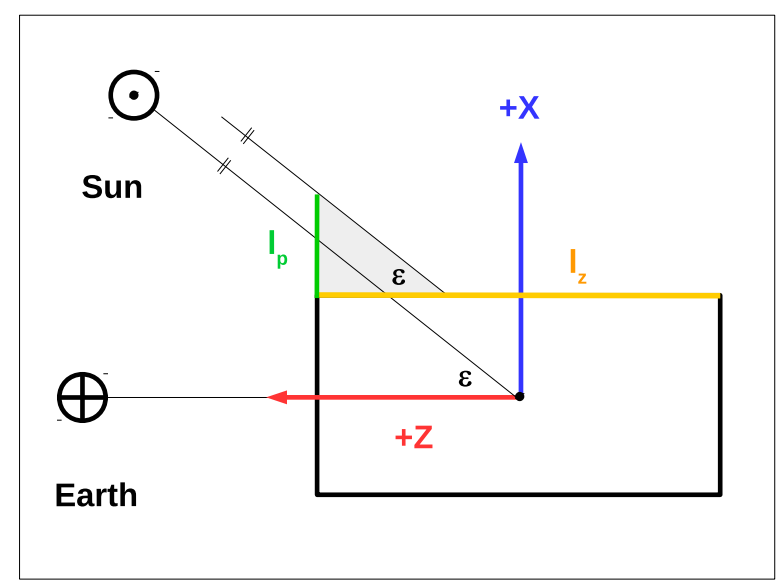

Fig. 3 Box-plate model of GIOVE-B. $l_{z}$ indicates the length of the satellite in $\mathrm{z}$-direction, $l_{p}$ the width of the plate. $\varepsilon$ stands for the Sun-satellite-Earth angle.

For the given plate width $l_{p} \approx 0.5 \mathrm{~m}$ and the length of the satellite in $z$-direction $l_{z} \approx 2.4 \mathrm{~m}$, this condition is fulfilled for $\varepsilon$ less than approximately $11.8^{\circ}$. It may be noted that this condition is only met when GIOVE-B is also in Earth shadow and is therefore of no practical relevance for the SRP modeling of this satellite. No shadowing effects occur for

$$
\varepsilon>\frac{\pi}{2} \text {. }
$$

For other values of $\varepsilon$, the fraction $s_{s h}$ of the $+x$-surface shadowed by the plate is given by

$$
s_{s h}=\frac{l_{p}}{l_{z}} \cdot \cot \varepsilon .
$$

With a shadowing factor of $s_{s h}=1$ for condition (5) and $s_{s h}=0$ for condition (6), Eqs. 3 and 4 can be rewritten to include the shadowing effects of the plate:

$$
\begin{gathered}
a_{C, s h}=a_{C} \quad-\frac{1}{2} s_{s h} \cdot a_{+x} \\
a_{S, s h}=a_{S} \quad+\frac{1}{2} s_{s h} \cdot a_{+x}
\end{gathered}
$$

with $a_{+x}=a_{C}-a_{S}$. Numerical values for the two different sets of parameterizations $\left(a_{C}, a_{S}\right)$ and $\left(a_{z}, a_{+x}\right)$ based on the satellite dimensions and the optical properties from Tab. 1 are listed in Tab. 2. Compared to Galileo $\operatorname{IOV}\left(a_{C}=+14.5 \mathrm{~nm} / \mathrm{s}^{2}, a_{S}=5.1 \mathrm{~nm} / \mathrm{s}^{2}\right)$, the stretch parameter $a_{S}$ has a negative value as the short side of the cuboid is pointing to the Earth whereas the long side is Earth-pointing for Galileo IOV.

\begin{tabular}{lclc}
\hline$a_{C}^{\alpha \delta}$ & $+15.8 \mathrm{~nm} / \mathrm{s}^{2}$ & $a_{C}^{\rho}$ & $+2.0 \mathrm{~nm} / \mathrm{s}^{2}$ \\
$a_{S}^{\alpha \delta}$ & $-2.3 \mathrm{~nm} / \mathrm{s}^{2}$ & $a_{S}^{\rho}$ & $-0.3 \mathrm{~nm} / \mathrm{s}^{2}$ \\
\hline$a_{z}^{\alpha \delta}$ & $13.5 \mathrm{~nm} / \mathrm{s}^{2}$ & $a_{z}^{\rho}$ & $1.7 \mathrm{~nm} / \mathrm{s}^{2}$ \\
$a_{+x}^{\alpha \delta}$ & $18.1 \mathrm{~nm} / \mathrm{s}^{2}$ & $a_{+x}^{\rho}$ & $2.3 \mathrm{~nm} / \mathrm{s}^{2}$ \\
\hline
\end{tabular}

Table 2 Characteristic accelerations for GIOVE-B based on the areas and optical properties of Tab. 1. The accelerations are given in two different parameterizations that can be converted with Eqs. 3 and 4.

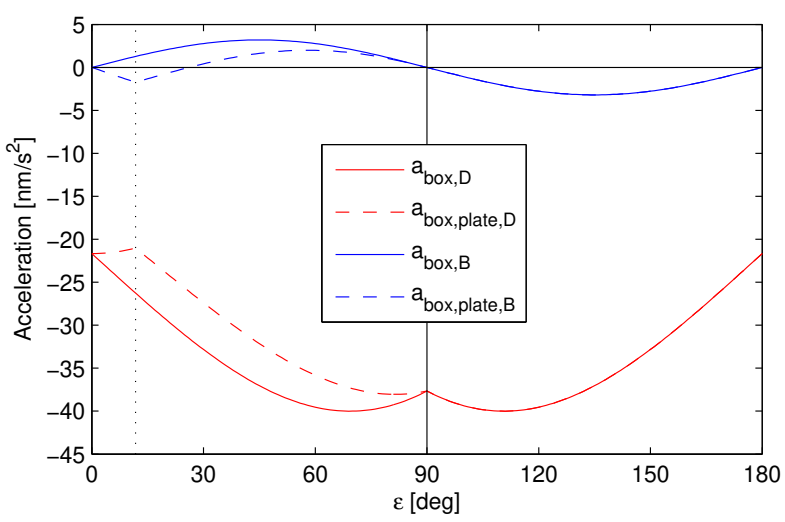

Fig. 4 Accelerations of the box-plate model from Tab. 3 (dashed lines) and a box model ignoring the shadowing effects of the plate (solid lines). The dotted line indicates the transition from full shadowing according to Eq. 5 to partial shadowing.

\subsection{Model Parameters}

The determination of the model parameters follows the approach of Montenbruck et al. (2014) based on numerically derived partial derivatives. The cuboidness parameter is fixed to $a_{C}=a_{C}^{\alpha \delta}+a_{C}^{\rho}=17.8 \mathrm{~nm} / \mathrm{s}^{2}$ as it is difficult to separate it from the contributions of the solar panels as well as to separate the $\alpha \delta$ and $\rho$ terms due to high correlations. The stretch parameter $a_{S}$ is estimated in a least squares adjustment. The direct radiation pressure parameters $D_{0}$ from 131 days of GIOVE-B precise orbit determination (see Sec. 3) without a priori model are used as observations in this parameter estimation. The three parameters describing the GIOVE-B box-plate model are listed in Tab. 3.

\begin{tabular}{lrll}
\hline Parameter & \multicolumn{2}{c}{ Numerical value } & Remark \\
\hline$a_{C}$ & +17.8 & $\mathrm{~nm} / \mathrm{s}^{2}$ & Table 2 \\
$a_{S}$ & -4.8 & $\mathrm{~nm} / \mathrm{s}^{2}$ & adjusted \\
$l_{p}$ & 0.5 & $\mathrm{~m}$ & assumed \\
\hline
\end{tabular}

Table 3 Parameters of the GIOVE-B box-plate model.

The accelerations of this box-plate model as well as a box model ignoring the shadowing effects are shown in Fig. 4. Differences only occur for $\varepsilon<90^{\circ}$ when the $+x$-surface is completely or partly shadowed. The discontinuity in the accelerations of the box-plate model at $11.8^{\circ}$ (indicated by a dotted line in Fig. 4) is related to the transition from complete shadowing of the $+x$-surface by the plate to increasing illumination. The impact of the plate amounts up to $3 \mathrm{~nm} / \mathrm{s}^{2}$ in $B$-direction and up to $5.5 \mathrm{~nm} / \mathrm{s}^{2}$ in $D$ direction.

\section{GNSS Data Processing}

The GNSS data analysis for determination and validation of the GIOVE-B box-plate model covers the second half of 2011 (day 197 - 327) due to the sparse tracking network before and unavailability of the PHM after that time period (switch from PHM to Rb on day 329/2011). Undifferenced GIOVE-B E1 and E5a code and phase observations of 26 


\begin{tabular}{ll}
\hline Parameter & Time resolution/additional information \\
\hline Station coordinates & 1 set per 3-day interval \\
Receiver clocks & epoch-wise \\
Differential code biases & per station and 1-day interval, 100 ns constraint \\
Ambiguities & fixed for GPS, float for GIOVE \\
Troposphere zenith delays & 2 h parameter spacing, mapped with wet GMF (Boehm et al., 2006) \\
Earth rotation parameters & polar motion offset/drift and LOD per 1-day interval \\
State vector & per 3-day interval \\
SRP parameters & $D_{0}, Y_{0}, B_{0}, B_{C}$, and $B_{S}$ for GPS and GIOVE \\
& tightly constrained constant, sine, and cosine accelerations \\
Satellite clocks & in along-track direction for GPS only \\
\hline
\end{tabular}

Table 4 Parameters estimated within the GNSS processing.

CONGO stations and GPS L1 and L2 code and phase observations of the CONGO as well as 61 additional IGS stations are simultaneously processed with the version 3.3.1 of the NAvigation Package for Earth Observation Satellites (NAPEOS, Springer, 2009). GPS observations are included to improve the quality of the parameters they have in common with the GIOVE parameter estimation (station coordinates, receiver clock parameters, troposphere parameters, and Earth rotation parameters). In order to further strengthen the orbital arcs of the sparse GIOVE tracking network, full 3-day solutions are computed.

A sampling rate of $5 \mathrm{~min}$, an elevation cut-off angle of $10^{\circ}$ and elevation-dependent weighting with $w=\sin \epsilon$ are applied. A priori hydrostatic troposphere zenith delays are computed with the global pressure and temperature model (GPT, Boehm et al., 2007) and mapped with the hydrostatic global mapping function (GMF, Boehm et al., 2006). GPS satellite antenna phase center offsets (PCOs) and variations $(\mathrm{PCVs}$ ) are taken from igs08.atx (Rebischung et al., 2012). GIOVE-B PCOs are taken from Zandbergen and Navarro (2008) but the PCVs are neglected due to lack of availability. GPS receiver antenna calibrations originate also from igs08.atx but the GPS L2 antenna calibrations are used for the GIOVE E5a signals. Nominal yaw steering attitude (Bar-Sever, 1996) is used for all GPS satellites as well as GIOVE-B.

In a first preprocessing step, outliers and cycle slips are detected. Stations with few observations or a bad data quality are rejected automatically. Based on a consecutive ambiguity float solution, GPS ambiguities are fixed to integers with the Melbourne-Wübbena approach (Melbourne, 1985; Wübbena, 1985) for baselines up to $6000 \mathrm{~km}$ with an average success rate of $97.7 \%$. These ambiguity parameters are kept fixed in the final parameter estimation run solving for the parameters listed in Tab. 4. Within this run, outliers are rejected in an iterative procedure.

\section{Model Validation}

Three solutions have been computed to study the impact of an a priori model on the GIOVE-B orbit and clock parameters: a reference solution without a priori model (none), a solution with an a priori model only taking into account the cuboid shape of the satellite (box, $s_{s h}=0$ for all $\varepsilon$ angles) and a solution with an a priori model which also takes into account the plate on the $+z$ side of GIOVE-B (box-plate). In order to allow for a physical interpretation, the sine and cosine terms of the CODE SRP parameters are referred to the orbit angle $\mu$ (orbit midnight - Earth satellite) instead of the argument of latitude (Montenbruck et al., 2014) and labeled as $B_{C}^{\star}$ and $B_{S}^{\star}$.
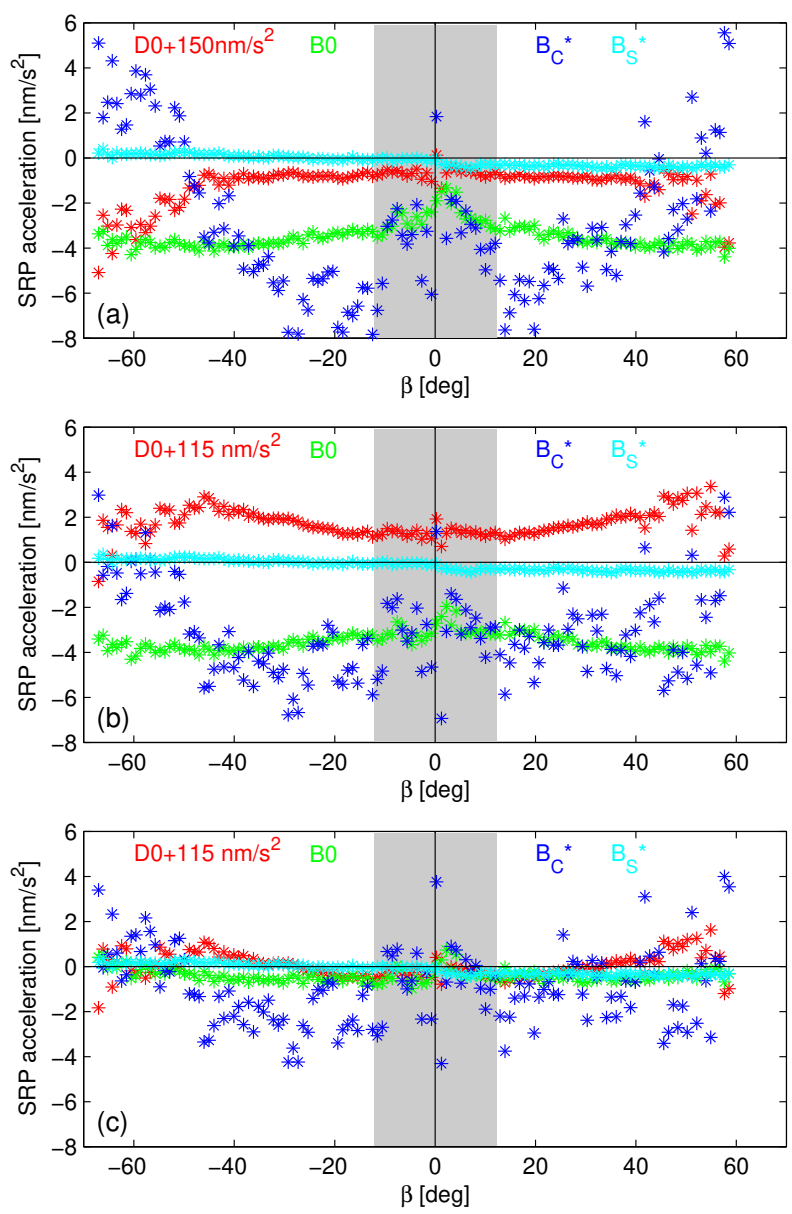

Fig. 5 SRP parameters estimated with different a priori models: (a) no a priori model; (b) box a priori model; (c) box-plate a priori model. The grey-shaded area indicates the eclipse period.

\subsection{Solar Radiation Pressure Parameters}

The estimated SRP parameters of the three different solutions are shown in Fig. 5 as a function of the elevation the 
Sun above the orbital plane $\beta$.

- The $D_{0}$ estimates are reduced by 38 and $36 \mathrm{~nm} / \mathrm{s}^{2}$ for the box and box-plate model, respectively, due to the mean $D_{0}$ contribution of the models, see Fig. 4.

- The $B_{0}$ estimates without and with box a priori model have a systematic bias of about $-3.5 \mathrm{~nm} / \mathrm{s}^{2}$. The bias is reduced to about $-0.5 \mathrm{~nm} / \mathrm{s}^{2}$ for the box-plate model.

- $B_{C}^{\star}$ varies by $14 \mathrm{~nm} / \mathrm{s}^{2}$ for the solution without a priori model. This variation is reduced to $10 \mathrm{~nm} / \mathrm{s}^{2}$ for the box model and $8 \mathrm{~nm} / \mathrm{s}^{2}$ for the box-plate model.

- The $B_{S}^{\star}$ estimates are very stable and do not depend on the a priori model. They only show a slight tilting w.r.t. the $\beta$-angle with a magnitude of $0.8 \mathrm{~nm} / \mathrm{s}^{2}$ over the whole $\beta$ range.

In general, the box-plate model reduces biases and scatter of the estimated SRP parameters through the refined modeling of the GIOVE-B spacecraft.

\subsection{Satellite Laser Ranging}

GIOVE-B is observed by the laser tracking stations of the International Laser Ranging Service (ILRS, Pearlman et al., 2002) on a regular basis. 4802 normal points are available for the analysis time interval. Only the middle day of the 3-day solutions is considered for the computation of the SLR residual statistics fixing the SLR station coordinates to SLRF2008 (Pavlis, 2009). Offset and standard deviation (STD) for the three solutions are given in Tab. 5 .

\begin{tabular}{lccc}
\hline a priori model & none & box & box-plate \\
\hline Offset $[\mathrm{cm}]$ & 10.7 & 8.3 & 0.8 \\
STD $[\mathrm{cm}]$ & 6.1 & 5.3 & 5.4 \\
RMS $[\mathrm{cm}]$ & 12.3 & 9.8 & 5.5 \\
\hline
\end{tabular}

Table 5 SLR validation of GIOVE-B microwave orbits for solutions with different a priori SRP models.

The STD improves by less than $1 \mathrm{~cm}$ when introducing an a priori model. This is a significantly different behavior compared to the Galileo IOV satellites: Montenbruck et al. (2014) report a reduction of the SLR STD by a factor of up to two when applying an a priori box model. This effect is attributed to the remaining banana-shaped signature of the SLR residuals plotted in Fig. 6. The a priori models only remove the slight rotation of this pattern in Fig. 6 resulting in only a small STD reduction. This systematics might be attributed to features of the satellite structure not considered with the simple box-plate model.

With a value of one decimeter, the SLR offset of the solution without a priori model is significantly larger than the noise of the SLR residuals. Introducing the a priori box model reduces the offset by about $20 \%$. When, in addition, considering the shadowing effects of the plate, the offset almost vanishes with a remaining value of less than one centimeter. The change in the SLR bias can, at least partly, be explained by a change of up to $5 \mathrm{~nm} / \mathrm{s}^{2}$ (Fig. 4) in the radial acceleration caused by the consideration of the shadowing effects. Even though the shadowing
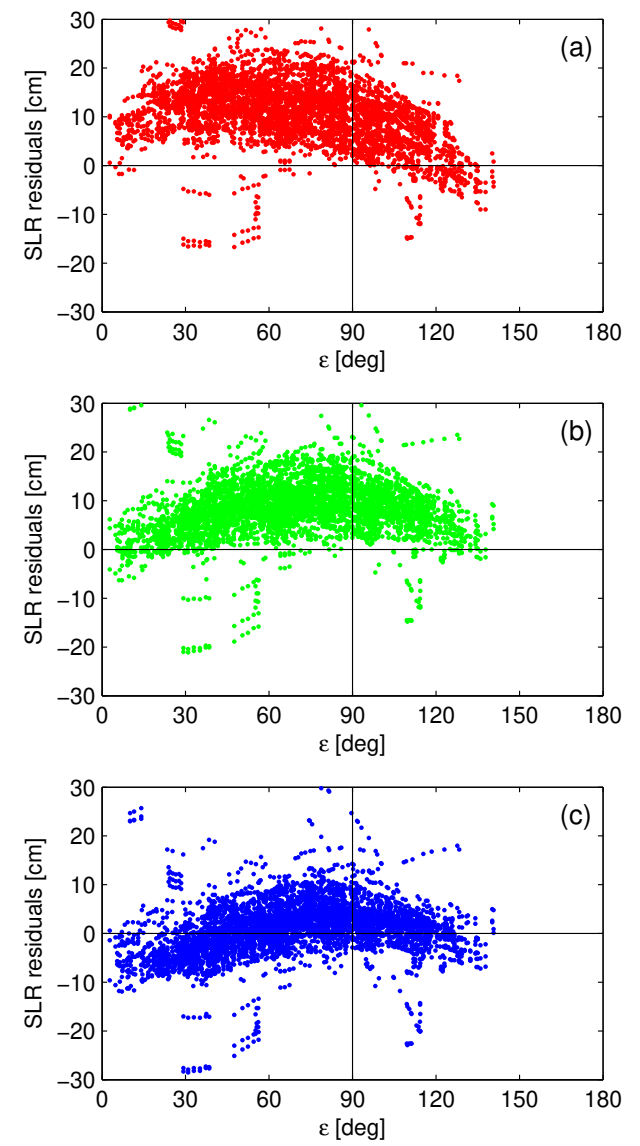

Fig. 6 GIOVE-B SLR residuals vs. Sun elongation $\varepsilon$ : (a) solution without a priori model, (b) solution with a priori box model, (c) solution with a priori box-plate model.

affects the computed acceleration only in the night hemisphere $\left(\approx 12.2^{\circ}<\varepsilon<90^{\circ}\right)$, the average radial acceleration over one revolution is also changed by about -0.5 to $-2.5 \mathrm{~nm} / \mathrm{s}^{2}$ depending on the actual $\beta$-angle. The consideration of shadowing effects is therefore similar to a slight increase in the gravitational acceleration and results in a corresponding increase of the orbital radius at given orbital period by $1-5 \mathrm{~cm}$. While this simplified consideration is not able to fully explain the observed SLR bias change it helps to understand it at least in a qualitative manner.

\subsection{Satellite Clock Parameters}

The highly stable PHM allows also to use satellite clock residuals as quality indicator for orbit modeling errors as these are mapped to the apparent clock parameters. As for the SLR residuals only the middle day is used for the clock analysis. For consistency, the GIOVE-B clock parameters estimated in the orbit determination are referred to a common reference clock (hydrogen maser at the IGS stations AMC2 located in Colorado Springs, USA or CRO1 located in Christiansted, Virgin Island). Day 271/2011 is excluded from all clock analysis due to a short transmission outage of GIOVE-B introducing a discontinuity in the clock time series.

Figure 7 shows the clock residuals of the three different solutions after removing a 2nd order polynomial. In contrast to Fig. 1 where a time series is shown, the Sun 

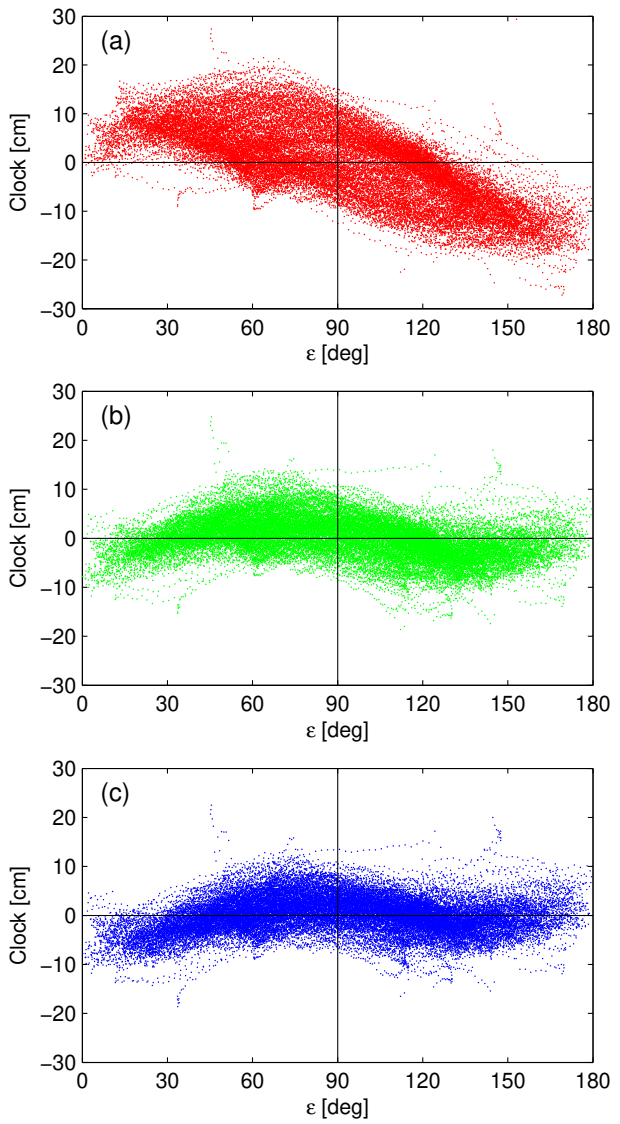

Fig. 7 GIOVE-B clock residuals vs. Sun elongation $\varepsilon$ : (a) solution without a priori model, (b) solution with a priori box model, (c) solution with a priori box-plate model.

elongation $\varepsilon$ is given on the $x$-axis of Fig. 7 to better illustrate the systematic variations of the full data set. The peak-to-peak amplitude of the clock residuals is reduced by a factor of two from $\pm 20 \mathrm{~cm}$ to $\pm 10 \mathrm{~cm}$ by the box and boxplate models. In contrast to the SLR residuals, the clock residuals allow for a continuous monitoring of the orbital errors including $\varepsilon$-angles larger than $140^{\circ}$. This additional information reveals a point symmetry of the clock residuals. As for the SLR residuals, a rotational component is removed by the a priori box and box-plate models but another systematic component remains in Fig. 7 (b) and (c). Nevertheless, the RMS of the clock residuals listed in Tab. 6 is reduced by a factor of about two for the solutions with box-plate model compared to the solution without a priori model.

\begin{tabular}{lccc}
\hline a priori model & none & box & box-plate \\
\hline RMS $[\mathrm{cm}]$ & 7.8 & 4.2 & 3.8 \\
\hline
\end{tabular}

Table 6 GIOVE-B clock residuals after removing a 2nd order polynomial for solutions with different a priori SRP models.

\subsection{Orbit Predictions}

A refined orbit modeling is expected to improve the performance of orbit predictions as well. Orbit differences between the observed orbit and $n$-day predictions (with

\begin{tabular}{lccccc}
\hline a priori model & \multicolumn{5}{c}{$n$-day orbit RMS [m] } \\
& 1-day & 2-day & 3-day & 4-day & 5-day \\
\hline none & 0.12 & 0.24 & 0.51 & 0.97 & 1.60 \\
box-plate & 0.12 & 0.24 & 0.51 & 0.97 & 1.62 \\
\hline
\end{tabular}

Table 7 GIOVE-B orbit prediction performance. 3D RMS values for orbit comparisons of the observed day w.r.t. a $n$-day prediction are given.

$n=1, \ldots, 5)$ are given in Table 7 . Surprisingly, there are essentially no differences between the solution without a priori model and the box-plate model. Applying no a priori model introduces a pronounced periodic pattern at the orbit frequency into the satellite trajectory as shown in Fig. 1. The amplitude of this periodic signal changes only slowly with changing $\beta$-angle. Within the maximum prediction interval of 5 days, these changes can be neglected. As a consequence, this periodic signal is also included in the orbit predictions resulting in almost the same performance as for the orbits applying the box-plate model. In view of this self-consistency of predicted and determined orbits, the overlap comparison cannot contribute to the assessment of the SRP modeling improvement.

\section{Summary and Conclusions}

Systematic patterns in GIOVE-B satellite laser ranging and clock residuals originate from not taking into account the shape of the satellite in the widely used CODE SRP model. By modeling the spacecraft with a simple box-plate model, variations and offsets in the estimated SRP parameters could be reduced. Whereas the STD of the SLR residuals is only slightly decreased by this model, the RMS of the clock residuals improves by a factor of two. The boxplate model largely removes the once-per-revolution clock error in Fig. 1 leaving a twice per revolution clock error with roughly half the peak-to-peak amplitude, see Fig. 8. The latter might be attributed to effects not yet considered in the orbit determination and remains subject to further studies.

The plate part of the box-plate model could be identified as the major source of the one decimeter SLR bias in the solution without a priori model. Considering the shadowing effects of the plate reduces the bias to less than one centimeter. It is worth to mention that no shadowing effects of the plate would have occurred if the plate would have been mounted on the $-x$ side of the satellite instead of the $+x$ side as the $-x$ side is never illuminated by the Sun due to the attitude law.

The simple box-plate model relies on coarse assumptions about the dimensions and optical properties of the spacecraft due to lack of more detailed information. A more sophisticated model could take into account:

- exact size and optical properties of the antenna panel

- distinction between solar cells and golden MLI to account for the different optical properties

- differences between $+z$ and $-z$ surface (antenna panel, LRA, front side of panel vs. launch adapter and backside of panel) 


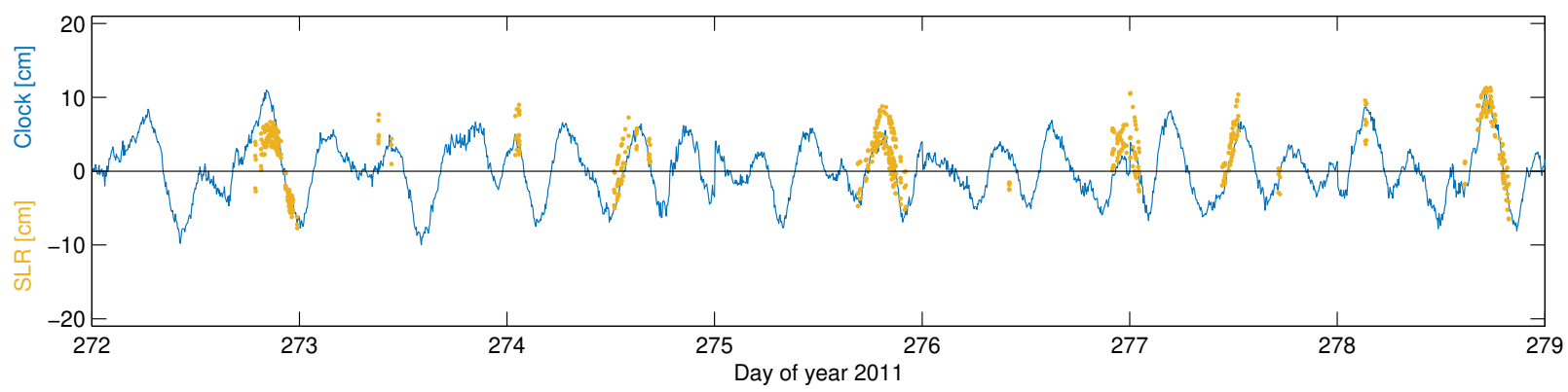

Fig. 8 Time series of GIOVE-B SLR and clock residuals with a priori box-plate model. The same time interval as in Fig. 1 is shown.

- smaller structures of the satellite (e.g., Earth sensors) and related shadowing effects

- thermal re-radiation.

However, detailed information from the manufacturer is required to further improve the model and perhaps remove systematic effects still visible in the SLR and clock residuals.

Acknowledgements We'd like to thank all institutions and individuals contributing to the CONGO and IGS networks, in particular the station operators and the data centers. The International Laser Ranging Service is acknowledged for a regular SLR tracking of GIOVE-B.

\section{References}

Bar-Sever, Y.E., 1996. A new model for GPS yaw attitude. J. Geod. 70, 714-723. Doi: 10.1007/BF00867149.

Benedicto, J., Gatti, G., Garutti, A., Paffet, J., Bradford, A., Jackson, C., Rooney, E., 2006. The triumph of GIOVE-A the first Galileo satellite. ESA Bulletin 127, 62-69.

Beutler, G., Brockmann, E., Gurtner, W., Hugentobler, U., Mervart, L., Rothacher, M., Verdun, A., 1994. Extended orbit modeling techniques at the CODE processing center of the international GPS service for Geodynamics (IGS): theory and initial results. Manuscripta Geod. 19, 367-386.

Boehm, J., Heinkelmann, R., Schuh, H., 2007. Short note: A global model of pressure and temperature for geodetic applications. J. Geod. 81, 679-683. Doi: 10.1007/s00190-0070135-3.

Boehm, J., Niell, A., Tregoning, P., Schuh, H., 2006. Global Mapping Function (GMF): A new empirical mapping function based on numerical weather model data. Geophys. Res. Lett. 33. Doi: 10.1029/2005GL025546.

Dell'Agnello, S., Monache, G.D., Currie, D., R. Vittori and, C.C., Garattini, M., Boni, A., Martini, M., Lops, C., Intaglietta, N., Tauraso, R., Arnold, D., Pearlman, M., Bianco, G., Zerbini, S., Maiello, M., Berardi, S., Porcelli, L., Alley, C., McGarry, J., Sciarretta, C., Luceri, V., Zagwodzki, T., 2011. Creation of the new industry-standard space test of laser retroreflectors for the GNSS and LAGEOS. Adv. Space Res. 47, 822-842. Doi: 10.1016/j.asr.2010.10.022.

Dow, J.M., Neilan, R.E., Rizos, C., 2009. The International GNSS Service in a changing landscape of Global Navigation Satellite Systems. J. Geod. 83, 191-198. Doi: 10.1007/s00190-008-0300-3.

Droz, F., Rochat, P., Wang, Q., 2010. Performance overview of space rubidium standards, in: 24th European Frequency and Time Forum.
ESA, 2011. GIOVE Experimentation Results: A Success Story. Technical Report SP-1320. European Space Agency. Noordwijk.

Gao, G., Akos, D., Walter, T., Enge, P., 2008. GIOVE-B on the air. Inside GNSS 3, 34-37.

García, Á.M., Píriz, R., Fernández, V., Navarro-Reyes, D., González, F., Hahn, J., 2008. GIOVE orbit and clock determination and prediction: Experimentation results, in: Proceedings of ENC GNSS 2008.

Giraud, J., Borrel, V., Crisci, M., 2009. Latest achievements in GIOVE signal and sensor station experimentations, in: ION GNSS 2009, pp. 3025-3036.

GPS World, 2014. The almanac - orbit data and ressources on active GNSS satellites. GPS World 25, 71-73.

Hackel, S., Steigenberger, P., Hugentobler, U., Uhlemann, M., Montenbruck, O., 2014. Galileo orbit determination using combined GNSS and SLR observations. GPS Sol. online first. Doi: $10.1007 / \mathrm{s} 10291-013-0361-5$.

Hidalgo, I., Tobias, G., Rodriguez, D., Binda, S., Gonzalez, F., Prieto, R., Tavella, P., Sesia, I., Cerretto, G., 2009. E-OSPF experimentation results in the frame of the GIOVE-M core infrastructure, in: Proceedings of the European Navigation Conference - Global Navigation Satellite Systems.

Kirchner, M., Schmidt, R., Vilzmann, J., 2009. Results of GIOVE data processing to allow evaluation of principal system performance drivers, in: Proceedings of the European Navigation Conference - Global Navigation Satellite Systems.

Kouba, J., Héroux, P., 2001. Precise point positioning using IGS orbit and clock products. GPS Sol. 5, 12-28.

Malik, M., Gatti, G., Alpe, V., Johansson, M., Kieffer, R., Robertson, G., 2009. GIOVE-B satellite \& payload overview, in: Proceedings of the European Navigation Conference Global Navigation Satellite Systems.

Melbourne, W.G., 1985. The case for ranging in GPS based geodetic systems, in: Goad, C. (Ed.), Proceedings of the First International Symposium on Precise Positioning with the Global Positioning System. U.S. Department of Commerce, Rockville, Maryland, pp. 373-386.

Montenbruck, O., Hauschild, A., Hessels, U., 2011. Characterization of GPS/GIOVE sensor stations in the CONGO network. GPS Sol. 15, 193-205. Doi: 10.1007/s10291-0100182-8.

Montenbruck, O., Steigenberger, P., Hugentobler, U., 2014. Enhanced solar radiation pressure modeling for Galileo satellites. J. Geod. online first. Doi: 10.1007/s00190-014-0774-0. Montenbruck, O., Steigenberger, P., Schönemann, E., Hauschild, A., Hugentobler, U., Dach, R., Becker, M., 2012. Flight characterization of new generation GNSS satellite clocks. Navigation, Journal of the Institute of Navigation 59, 291-302. 
Ostillio, A., Johansson, M., Hannes, D., Malik, M., Waller, A.R.P., Belloni, M., Droz, F., Mosset, P., 2009. Passive Hydrogen Maser (PHM): the heart of the Galileo navigation payload, in: Proceedings of the European Navigation Conference - Global Navigation Satellite Systems.

Pavlis, E., 2009. SLRF2008: The ILRS reference frame for SLR POD contributed to ITRF2008, in: 2009 Ocean Surface Topography Science Team Meeting, Seattle.

Pearlman, M., Degnan, J., Bosworth, J., 2002. The International Laser Ranging Service. Adv. Space Res. 30, 125-143. Doi: 10.1016/S0273-1177(02)00277-6.

Prange, L., Dach, R., Lutz, S., Schaer, S., Jäggi, A., 2014. The CODE MGEX orbit and clock solution, in: Willis, P. (Ed.), IAG Potsdam 2013 Proceedings, Springer. Accepted for publication.

Rebischung, P., Griffiths, J., Ray, J., Schmid, R., Collilieux, X., Garayt, B., 2012. IGS08: the IGS realization of ITRF2008. GPS Sol. 16, 483-494. Doi: 10.1007/s10291-011-0248-2.

Robertson, G., Kieffer, R., Malik, M., Gatti, G., Alpe, V., Johansson, M., 2009. GIOVE-B satellite design and performance validation, in: ION GNSS 2009, pp. 3008-3016.

Rodriguez-Solano, C.J., Hugentobler, U., Steigenberger, P., 2012. Adjustable box-wing model for solar radiation pressure impacting GPS satellites. Adv. Space Res. 49, 11131128. Doi: 10.1016/j.asr.2012.01.016.

Schönemann, E., Springer, T., Otten, M., Becker, M., Dow, J., 2007. GIOVE-A precise orbit determination from microwave and satellite laser ranging data - first perspectives for the Galileo constellation and its scientific use, in: Proceedings of the First Colloquium on Scientific and Fundamental Aspects of the Galileo Programme 2007.

Springer, T., 2009. NAPEOS Mathematical Models and Algorithms. Technical Report DOPS-SYS-TN-0100-OPS-GN. ESA/ESOC, Darmstadt.

Steigenberger, P., Hugentobler, U., Loyer, S., Perosanz, F., Prange, L., Dach, R., Uhlemann, M., Gendt, G., Montenbruck, O., 2014. Galileo orbit and clock quality of the IGS multi-GNSS experiment. Adv. Space Res. online first. Doi: $10.1016 /$ j.asr.2014.06.030.

Steigenberger, P., Hugentobler, U., Montenbruck, O., Hauschild, A., 2011. Precise orbit determination of GIOVEB based on the CONGO network. J. Geod. 85, 357-365. Doi: 10.1007/s00190-011-0443-5.

Svehla, D., Schönemann, E., Escobar, D., Springer, T., 2010. Complete relativistic modelling of the GIOVE-B clock parameters and its impact on POD, track-to-track ambiguity resolution and precise timing applications, in: IGS Analysis Center Workshop 2010, Newcastle, UK.

Uhlemann, M., Gendt, G., Ramatschi, M., Deng, Z., 2014. GFZ global multi-GNSS network and data processing results, in: Willis, P. (Ed.), IAG Potsdam 2013 Proceedings, Springer. Accepted for publication.

Wübbena, G., 1985. Software developments for geodetic positioning with GPS using TI-4100 code and carrier measurements, in: Goad, C. (Ed.), Proceedings of the First International Symposium on Precise Positioning with the Global Positioning System. U.S. Department of Commerce, Rockville, Maryland, pp. 403-412.

Zandbergen, R., Navarro, D., 2008. Specification of Galileo and GIOVE space segment properties relevant for satellite laser ranging. Technical Report ESA-EUING-TN/10206, iss. 3.2, 08/05/2008. ESA/ESOC, Darmstadt. 